\title{
Interpretation of EUV emissions observed by Mills et al.
}

\author{
A.V. Phelps ${ }^{1, a}$ and J. Clementson ${ }^{2}$ \\ 1 JILA, National Institute of Standards and Technology and University of Colorado Boulder, 80309-0440 Boulder, CO, USA \\ 2 Lawrence Livermore National Laboratory, 94551-0808 Livermore, CA, USA
}

Received 15 February 2012

Published online 17 May 2012

(C) The Author(s) 2012. This article is published with open access at Springerlink.com

\begin{abstract}
An explanation of the so-called hydrino continuum emissions proposed by Mills and Lu, most recently in [Eur. Phys. J. D 64, 65 (2011)], is presented using conventional atomic, plasma, and discharge physics. It is argued that the observed EUV emissions during their pulsed discharges originate from transitions in ions sputtered or evaporated from the electrodes. Such an interpretation removes their justification for the introduction of hydrino particles.
\end{abstract}

Recently, Mills and Lu have published extreme ultraviolet (EUV) spectra from pulsed discharges, which they claim prove the existence of so-called hydrino atoms (hydrogen atoms with states having a fractional principal quantum number) [1-4]. The discharges were produced using various fill gases and cathode materials. The observed spectra could not be explained by Mills et al. using conventional physics, which allowed them to interpret the data as evidence for their hydrino model. In the present paper, it is argued that the observed spectra are, in fact, emissions from low charge-state ions sputtered or evaporated from the electrodes plus some impurity ion emission. The continuum appearance is explained as being made up of densely spaced lines that are not fully resolved. Specific points are:

Mills et al. claim [1-4] there are no differences in the observed discharge spectra when different electrode materials are used. In Figures 1 through 3 spectra have been digitized $^{1}$ from plots of Mills et al. [1-4] for emission observed with cathodes made of tungsten (W), molybdenum (Mo), and tantalum (Ta). These traces show instead that the spectra differ considerably with electrode material. The dependence on material is especially clear in Figures $1 \mathrm{a}$ and $1 \mathrm{~b}$, where discharges with $\mathrm{W}$ and Mo cathodes using a hydrogen $\left(\mathrm{H}_{2}\right)$ fill gas are compared.

Mills et al. [1-4] further state that the observed emission cannot be explained as line emission by atoms or ions from the metal electrodes. Here it is shown that much of

\footnotetext{
${ }^{a}$ e-mail: avp@jila.colorado.edu

${ }^{1}$ Because requested original data tabulations have not been made available, the data shown for Mills et al. are obtained by digitizing figures selected for minimum diffusiveness and non-overlapping curves from the referenced publications and "secured" preprint files. Thus, for the data of reference [4] in Figures 1 and 2, the pixel width seen by the digitizer software is larger than the nominal spectrometer resolution of $0.05 \mathrm{~nm}$.
}

the emission can indeed be explained as line radiation from low charge states of sputtered or evaporated high-Z metal ions. In Figure 1a the spectrum of Mills et al. is plotted together with W spectra observed by Clementson et al. [5] ${ }^{2}$ from an electron beam ion trap (EBIT). The data were acquired using a grazing-incidence instrument with a resolution of $0.04 \mathrm{~nm}$ full width half maximum (FWHM) at an EBIT electron-beam energy of $150 \pm 10 \mathrm{eV}$. This energy is high enough to create $\mathrm{W}$ ions with charge states up to $8+[6]$. Very similar spectra at lower resolution (not shown) have been obtained by evaporating tungsten into a tokamak device $[7]^{3}$ and selecting an early stage of plasma development. Other observations [8-10] of W ion spectra in this wavelength region were obtained under less well defined conditions or later stages of plasma development. As expected, no hydrogen emission is observed in the spectral region of these experiments.

Comparison of the experimental spectra from Mills et al. [1-4] with calculations of oxygen (O) ion emission in Figure 1a supports their identification of the lines at 15.0, 17.3 , and $19.3 \mathrm{~nm}$ as $\mathrm{O}$ VI and $\mathrm{O} \mathrm{V}$ lines ${ }^{4}$. The calculated relative intensities of $\mathrm{O}$ ion emission are obtained using a coronal equilibrium model ${ }^{5}$. The observation of lines of the

\footnotetext{
2 These spectra are free from C, O and other lighter impurity ions because of evaporative cooling with the heavy $\mathrm{W}$ ions.

${ }^{3}$ A laser induced blow-off technique is used to inject $\mathrm{W}$ atoms. The EUV is recorded during plasma buildup to emphasize lower degrees of ionization.

${ }^{4}$ If $\mathrm{O}$ or $\mathrm{N}$ were sputtered or evaporated from the surface as proposed in reference [1], these gases should clean-up as the spectral scan was recorded. Also, significant quantities of surface impurities should be detectable as a burst of these gases in the residual gas detector of the vacuum system.

5 The plasma is assumed to be in steady-state coronal equilibrium at an electron temperature of $25 \mathrm{eV}$ and an electron density of $10^{16} \mathrm{~cm}^{-3}$. The calculations utilized the Flexible Atomic Code described in [11].
} 


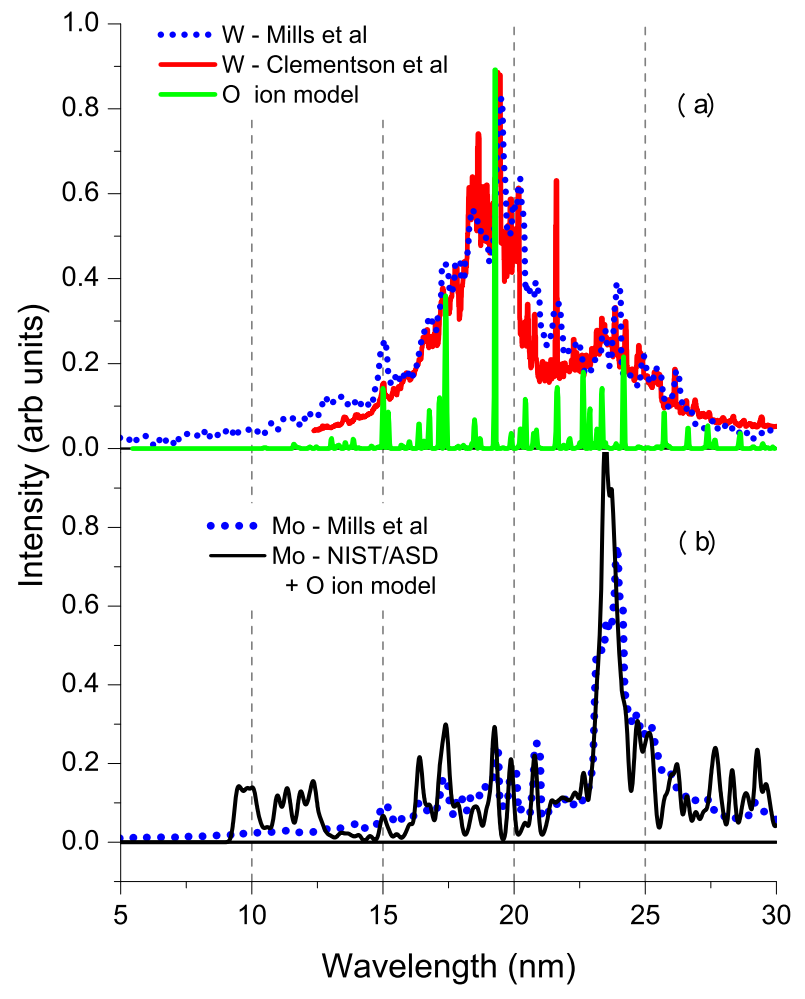

Fig. 1. (Color online) EUV emission data from pulsed discharge experiments with tungsten (a) and molybdenum (b) electrodes. The dotted curves are from figures $12 \mathrm{a}$ and $4 \mathrm{a}$ of [4].

O VI ion [1] shows the presence of electrons with energies above the $113.9 \mathrm{eV}$ production threshold and is consistent with the presence of the high energy electrons required to produce the $\mathrm{W}$ ion spectra cited above.

The barely resolved structure in the Mills et al. [2-4] data of Figure 1a at wavelengths between 20 and $22 \mathrm{~nm}$ shows at least four coincidences between lines (or groups of lines) as observed using the EBIT device with a pure $\mathrm{W}$ plasma $[5]^{2}$. These coincidences are confirmed by high resolution scans (not shown) with the EBIT source $[5]^{2}$ operating at an electron beam energy of $128 \pm 10 \mathrm{eV}$. In particular, the line at $21.6 \mathrm{~nm}$, dominating the EBIT data, is a strong line of W VII [12-14], while the calculated $\mathrm{O} \mathrm{V}$ line intensity at nearly the same wavelength is predicted to be relatively weak ${ }^{5}$. Because tabulated spectra of W VIII and W IX are not available [12,13,15], these tests cannot be made on the shorter wavelength portion of the data. The present line identifications contradict the claim of Mills et al. [2-4] that no lines from the tungsten electrode material are observed.

As a second example, Figure 1b shows the similarities of spectral distribution of intensity for the pulsed discharge of reference [4] using Mo electrodes to the spectral distribution for an empirically assumed plasma of $4 \%$ Mo VII ions, $14 \%$ Mo VIII ions and $82 \%$ Mo IX ions as calculated using the relative line intensities from the NIST/ASD tables $[12,13$. The thresholds for production of these Mo spectra [16], at 68.8, 125.7 and $143.6 \mathrm{eV}$, are roughly equal to the threshold for the $\mathrm{W}^{8+}$ ion $[6]$ of $141 \mathrm{eV}$. The calculated curve also includes the calculated O ion spectrum ${ }^{5}$ of Figure 1a multiplied by a fitting constant. No correction to the model spectra has been made for instrumental sensitivity. The plasma is assumed spatially uniform, although its true state is unknown. Freefree and free-bound emission are estimated to be small. The calculation assumes a Gaussian line profile with the effective instrumental width of $0.12 \mathrm{~nm}$ (FWHM) chosen to approximately fit the structure in the data of Mills et al. [4] obtained using a 600 line/mm grating. Differences between experimental and calculated spectra are comparable with differences in the various experimental spectra of Mills et al. [2-4] for Mo electrodes. Obviously, more direct experiments are needed to confirm the calculated Mo spectral distribution. In summary, the consistency of the comparisons in Figure 1 shows that the EUV spectra in references [1-4] are characteristic of the respective electrode materials plus an oxygen impurity.

The data of Figure 1b also show that in contrast to the approximate agreement between experiment and calculation from 15 to $30 \mathrm{~nm}$, the predicted band of emission from 9 to $12 \mathrm{~nm}$ is absent from experiment. This disagreement may mean the relative intensities of lines emitted by Mo IX obtained from spark discharge experiments $[12,13]$ are not applicable to the pulsed discharges of references [1-4] because of a deficiency of electrons with energies above the $\approx 110 \mathrm{eV}$ threshold energy $[12,13]$. In this case, the absence of emission at short wavelengths is simply a matter of the metal ion properties. Alternatively, it could mean that the near absence of emission below $10 \mathrm{~nm}$ reported in references [1-4] for their electrode materials and gas fills is the result of loss of spectrometer sensitivity. In view of the reasonableness of these explanations, further experiments would be required in order to justify citing the weak emission at below about $10 \mathrm{~nm}$ as supporting the hydrino model [1].

The data of Figure 2, shown on an expanded wavelength scale, are used to address more directly the cause of the pseudo-continuum observed in the data of Mills et al. [1-4] for their 600 line $/ \mathrm{mm}$ grating. The low resolution curve designated NIST/ASD of Figure 2 is an expanded portion of the calculated curve of Figure $1 \mathrm{~b}$ for Mo ions, while the high resolution curve shows the same spectrum calculated assuming Doppler profiles corresponding to an ion temperature of $10 \mathrm{eV}$. Although some of the broadening and shift of the emission peaks from reference [4] may be the result of digitizing errors, approximate fitting of these data requires that the effective instrumental line width (including the effects of line wings, variations with wavelength, ghosts, etc.) is nearer $0.15 \mathrm{~nm}$ (FWHM) than the stated $0.05 \mathrm{~nm}$ resolution. Fitting of their data for a 1200 line/mm grating (not shown) requires an effective resolution of about $0.07 \mathrm{~nm}$ (FWHM). From the similarity of the calculated low resolution curve and experiment, it is concluded that the pseudo-continuum discussed in references [1-4] is the result of overlapping and unresolved spectral lines. This interpretation is supported by experimental observations $[5]^{2},[9]$ and 


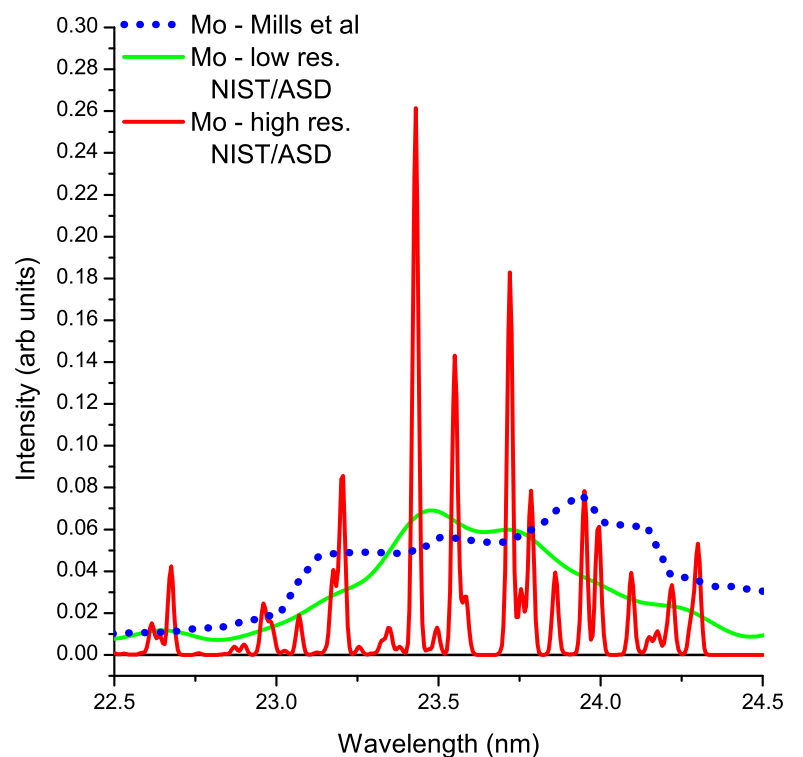

Fig. 2. (Color online) EUV emission data for Mo ions. The curve from Mills et al. for Mo is from figure 4a of [4]. The low and high resolution curves for Mo are calculated from NIST/ASD tables [12] assuming Gaussian line profiles with widths (FWHM) of 0.15 and $0.01 \mathrm{~nm}$, respectively.

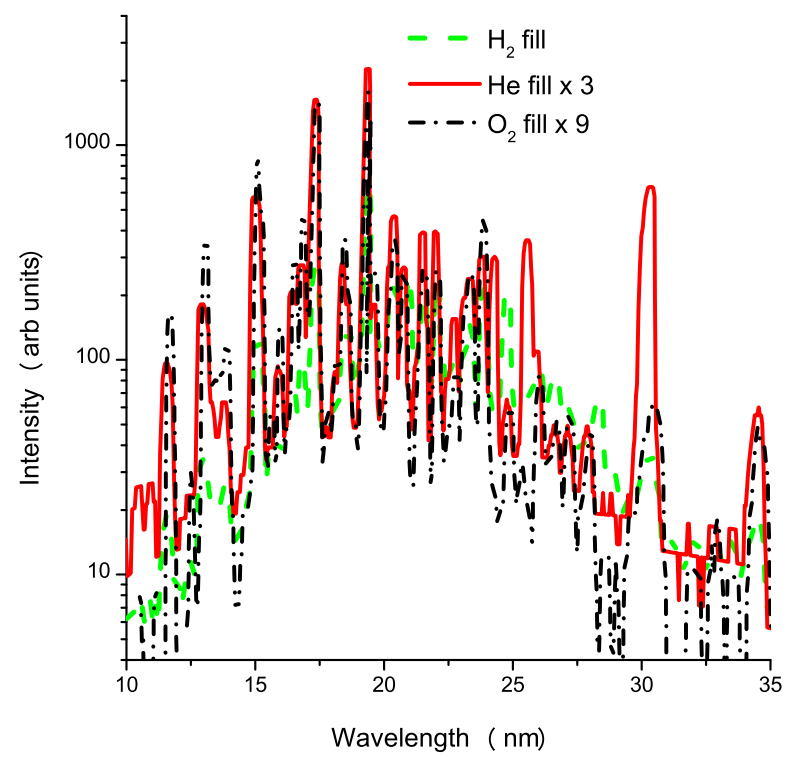

Fig. 3. (Color online) Spectral scans for Ta electrodes with $\mathrm{H}_{2}$, $\mathrm{He}$, and $\mathrm{O}_{2}$ fill gases from figures $3 \mathrm{e}, 3 \mathrm{c}$, and $3 \mathrm{a}$, respectively, of [1]. For the $\mathrm{He}$ and $\mathrm{O}_{2}$ gas fills, a small background decreasing with wavelength (such as expected for scattered light) and $\leq 10 \%$ of the peak pseudo-continuum at $20 \mathrm{~nm}$ is subtracted from the original data.

calculations [17] of "quasicontinuum" emission which result from overlapping lines of highly charged $\mathrm{W}$ ions. It is therefore argued that the observed pseudo-continua are not the continua predicted by the hydrino model [1].

The plots of Figure 3 are used to address the claim of reference [1] that the spectral scans obtained when using a
$\mathrm{H}_{2}$ fill gas show strong continua while those for other gas fills show no continua. These logarithmic plots show that the shapes of the pseudo-continua portions of the spectral scans for $\mathrm{He}$ and $\mathrm{O}_{2}$ gas fills are the same as for the $\mathrm{H}_{2}$ gas fill. Data for Ta electrodes and for a $\mathrm{N}_{2}$ fill [1] and for a Ar fill [2] are consistent with the plots of Figure 3, but because of a high noise level or limited wavelength range are not shown. The similarity of these data plots are expected from the model in which the pseudo-continua are produced by overlapping lines emitted by ions of the Ta electrodes. Compilations of analyzed spectral data for the moderate degree of ionization expected, e.g., Ta VIII, are not available $[12,13]$. The lines from $\mathrm{O}$ impurity ions are not expected to scale with the densities of Ta ions and so are not suitable for normalization of the pseudocontinuum as effectively done in figure 3 of [1]. The apparently significantly lower Ta ion densities obtained with the $\mathrm{He}$ and $\mathrm{O}_{2}$ gas fills than with the $\mathrm{H}_{2}$ fill may be explained by lower sputtering and/or evaporation rates for these gas fills. Thus, it is argued that the differences in metal vapor densities and EUV emission can be caused by different discharge conditions ${ }^{6}$ and provide no evidence that the existence of the pseudo-continua is dependent on the presence of hydrogen. Similar arguments apply to the data for $\mathrm{H}_{2} / \mathrm{He}$ mixtures with $\mathrm{W}$ electrodes shown in figure 9 of reference [2]. Obviously, independent determinations of metal ion densities in the pulsed discharges are needed.

Finally, the transient emission data shown in figures 4 through 6 of reference [1] are consistent with the emission model discussed above and do not require a hydrino-based model. Thus, the early peaking of the $\mathrm{O}$ ion emission in figure 5 relative to time of emission of the pseudo-continuum in figure 4 is expected from the sputtering or evaporation of a surface layer of oxygen ${ }^{4}$ prior to the production and multistage ionization of the electrode metal. The delayed emission from ionized electrode material has been observed $[18,19]$ for somewhat similar pulsed discharges ${ }^{6}$.

In summary, the EUV data presented in references [1-4] can be explained in terms of conventional collision, radiation, and gas discharge processes and do not support the hydrino hypothesis [1]. A Comment on the more general aspects of the hydrino theory has recently been published by Lawler and Goebel [20].

The work of A.V.P. was supported in part by JILA. The work of J.C. was preformed under the auspices of the United States Department of Energy by Lawrence Livermore National Laboratory under Contract No. DE-AC52-07NA-27344. A.V.P. thanks B.W. Bach, B.W. Bach Jr., P. Beiersdorfer, S. Fuelling, and E. Wilkinson for helpful discussions of the properties of

\footnotetext{
${ }^{6}$ Because sputering yields are expected to vary rapidly with ion mass and energy [21], the evaluation of sputtering as the source of ions requires detailed information as to the transient behavior of cathode emission modes, discharge electric fields, etc. in the pulsed discharges $[18,19]$. Similarly, estimates of electrode evaporation require extensive data on cathode bombardment, thermal response, etc. No transient voltage and current waveforms are provided in reference [1], although Bykanov [3] estimates some average discharge parameters.
} 
EUV spectrometers. He also thanks A. Gallagher for helpful discussions and reviewing the manuscript.

\section{References}

1. R.L. Mills, Y. Lu, Eur. Phys. J. D 64, 65 (2011)

2. R.L. Mills, Y. Lu, Int. J. Hydrogen Energy 35, 8446 (2010)

3. A. Bykanov, private communication (2011), http://www . blacklightpower.com/pdf/GEN3_Harvard.pdf

4. R.L. Mills, R. Booker, Y. Lu, private communication (2011), http://www.blacklightpower.com/papers/ SoftXray.pdf

5. J. Clementson, T. Lennartsson, P. Beiersdorfer, private communication (2011)

6. A.E. Kramida, J. Reader, At. Data Nucl. Data Tables 92, $457(2006)$

7. G. Veres, J.S. Bakos, B. Kardon, J. Quant. Spectrosc. Radiat. Transfer 56, 295 (1996)

8. S.G. Vasenin, N.I. Arkhipov, V.P. Bakhtin, S.M. Kurkin, V.M. Safronov, D.A. Toporkov, H. Wuerz, A.M. Zhitlukhin, Problems of Atomic Science and Technology (Kharkov, Ukraine, 2000), Vol. 6, p. 97, http://vant. kipt.kharkov.ua/ARTICLE/VANT_2000_6/article_2000_ 6_97.pdf. This reference was brought to our attention by S. Fuelling

9. T. Pütterich, R. Neu, R. Dux, A.D. Whiteford, M.G. O'Mullane, ASDEX Upgrade Team, Plasma Phys. Control. Fusion 50, 085016 (2008)

10. J. Clementson, P. Beiersdorfer, E.W. Magee, H.S. McLean, R.D. Wood, J. Phys. B 43, 144009 (2010)

11. M.F. Gu, Can. J. Phys. 86, 675 (2008)
12. Yu. Ralchenko, A.E. Kramida, J. Reader, NIST ASD Team, NIST Atomic Spectra Database (verion 4.1.0) [Online] (National Institute of Standards and Technology, Gaithersburg, MD, 2011), http://physics.nist.gov/ asd. The relative line intensities for Mo used in the model of Figure 1b are those listed in this compilation. These tables do not include the lines for W V, W VI, W VIII and W IX spectra in the wavelength region of current interest.

13. T. Shirai, J. Sugar, A. Musgrove, W.L. Wiese, Spectral data highly ionized atoms: $\mathrm{Ti}, \mathrm{V}, \mathrm{Cr}, \mathrm{Mn}, \mathrm{Fe}, \mathrm{Co}, \mathrm{Ni}, \mathrm{Cu}$, Kr, and Mo (American Inst. Phys., Melville, 2000), p. 477

14. J. Sugar, V. Kaufman, Phys. Rev. A 12, 994 (1975)

15. A.E. Kramida, T. Shirai, At. Data Nucl. Data Tables 95, $305(2009)$

16. T. Shirai, Y. Nakai, K. Ozawa, K. Ishii, J. Sugar, K. Mori, J. Phys. Chem. Ref. Data 16, 327 (1987)

17. C.S. Harte, C. Suzuki, T. Kato, H.A. Sakaue, D. Kato, K. Sato, N. Tamura, S. Sudo, R. D'Arcy, E. Sokell, J. White, G. O'Sullivan, J. Phys. B 43, 205004 (2010)

18. P. Felsner, J. Christiansen, K. Frank, M. Stetter, IEEE Trans. Plasma Sci. 23, 305 (1995)

19. Yu.D. Korolev, K. Frank, IEEE Trans. Plasma Sci. 27, 1525 (1999)

20. J.E. Lawler, C.J. Goebel, Eur. Phys. J. D 66, 29 (2012)

21. Y. Yamamura, H. Tawara, At. Nucl. Data Tables 62, 149 (1996)

Open Access This is an open access article distributed under the terms of the Creative Commons Attribution License (http://creativecommons.org/licenses/by/3.0), which permits unrestricted use, distribution, and reproduction in any medium, provided the original work is properly cited. 